KCL-PH-TH/2014-9

\title{
How well do we understand the thermal history of the Universe? Implications of the recent BICEP2 data
}

\author{
Mairi Sakellariadou* \\ Department of Physics, King's College London, University of London, Strand WC2R 2LS, London, U.K.
}

\begin{abstract}
Applying Grand Unified Theories in the context of the early Universe, cosmic strings are an unavoidable partner of the inflaton field. To render such models compatible with the CMB temperature anisotropies, the inflationary scale has to be less than $10^{15} \mathrm{GeV}$, a value which is inconsistent with the high inflationary scale deduced from the tensor-to-scalar ratio announced from the BICEP2 collaboration. Some questions may be consequently raised about our understanding of the thermal history of the Universe.
\end{abstract}

PACS numbers: 98.80.-k,98.80.Cq

Accepting the validity of Grand Unified Theories (GUTs) leads to the conclusion that the Universe, in the earliest stages of its evolution, was hotter and consequently in a more symmetric state. Subsequently, in the expansion process (Hubble's law), its temperature dropped and the Universe underwent a series of phase transitions followed by spontaneous breakdown of symmetries. Topological defects could then be left as remnants. In the context of (local) gauge theories - global theories, being rather unphysical, will not be discussed here - cosmic strings [ $1-3]$ have always been considered as the only type of (local) topological defects compatible with the observed Universe.

To dilute any undesired topological defects - domain walls, monopoles or textures — a stage of cosmological inflation [4 $[\underline{6}]$ is usually imposed. Such an inflationary era solves (by construction) the horizon and flatness problems, while as a bonus, it provides a mechanism for the origin of adiabatic Gaussian fluctuations, in consistency with the measured Cosmic Microwave Background (CMB) temperature anisotropies. Hence, cosmological inflation can account for the observed large-scale structure, within the framework of the theory of gravitational instability.

In this spirit, one has to examine defect formation within the series of phase transitions from a large GUT gauge group $\mathrm{G}_{\mathrm{GUT}}$ down to the Standard Model $(\mathrm{SM})$ group $\mathrm{G}_{\mathrm{SM}}=\mathrm{SU}(3) \times \mathrm{SU}(2) \times \mathrm{U}(1)$, with $\mathrm{G}_{\mathrm{SM}} \subset \mathrm{G}_{\mathrm{GUT}}$, and then postulate an inflationary era at the end of the (last) formation of any undesired defects.

In the context of GUTs, it has been commonly suggested the type of hybrid inflation [7] realised within a scheme

$$
\mathrm{G} \stackrel{M_{\mathrm{GUF}}}{\longrightarrow} \mathrm{H}_{1} \underset{\Phi_{+} \Phi_{-}}{\stackrel{M_{\text {infl }}}{\longrightarrow}} \mathrm{H}_{2} \rightarrow \mathrm{G}_{\mathrm{SM}}
$$

where $\Phi_{+}, \Phi_{-}$is a pair of GUT Higgs superfields in non-trivial complex conjugate representations, which lower the rank of the group by one unit when acquiring a non-zero vacuum expectation value. The inflationary stage takes place at the beginning of the symmetry breaking $\mathrm{H}_{1} \stackrel{M_{\text {infl }}}{\longrightarrow} \mathrm{H}_{2}$ and is based on a globally supersymmetric renormalisable superpotential $W$. Note that $\mathrm{G}$ stands here for either $\mathrm{G}_{\mathrm{GUT}}$ or $\mathrm{G}_{\mathrm{GUT}} \times \mathrm{U}(1)$.

Performing a rather exhaustive investigation, it has been shown in Ref. [8] that cosmic strings are generically formed at the end of hybrid inflation. Hence, provided Grand Unified Theories is a valid hypothesis, cosmic strings are expected to be left behind, as relics of an earlier more symmetric phase. Their cosmological consequences - and therefore their observational fingerprints - are mainly based on their gravitational interactions, which essentially depend on their linear mass density $\mu \sim T^{2}$ (with $T$ denoting the temperature of the phase transition during which strings were formed), which is intimately related to the scale of inflation.

Switching gears and moving to M-theory, in which branes of various dimensions are embedded in a higher dimensional space, one can still build a (brane) inflationary scenario, as the outcome of brane interactions. Within IIB string theory there are two classes of brane inflation models: D3/D7 inflation $9-11]^{1}$ and brane-antibrane inflation with D3/D3 [13] being the most studied example. Such brane inflation models generically accommodate [14, 15] cosmic superstrings [16, 17], which will then play the rôle of their field theoretic analogues.

\footnotetext{
*mairi.sakellariadou@kcl.ac.uk

${ }^{1}$ It is worth noting the caveat discussed in Ref. [12].
} 
Assuming the validity of the above discussion, one would expect [18] that cosmic (super)strings are a legitimate partner of the inflaton field, both seeding the initial density fluctuations which lead to the measured CMB temperature anisotropies. Hence, one may consider [18]

$$
C_{\ell}=\alpha C_{\ell}^{\mathrm{I}}+(1-\alpha) C_{\ell}^{\mathrm{S}}
$$

where $C_{\ell}^{\mathrm{I}}$ and $C_{\ell}^{\mathrm{s}}$ stand for the (COBE normalised) Legendre coefficients due to adiabatic inflaton fluctuations and those stemming from a cosmic (super)string network, respectively; the coefficient $\alpha$ is a free parameter expressing the relative amplitude for the two contributions.

Such theoretical constructions have to be tested against observational data and astrophysical measurements, and this has been the aim of various studies, in particular with respect to the CMB data [19, 20]. Let us leave aside the precise predictions of a given model, and summarise the generic predictions of each class of models (strings, and inflation), which turn out to be intrinsically different for reasons which are known for about two decades.

Cosmic (super)string models lead to isocurvature density perturbations - the total density perturbation vanishes, whilst the density perturbation of individual particle species does not. More importantly, perturbations are generated continuously; they evolve according to inhomogeneous linear perturbation equations, with the energy momentum tensor of strings being determined by their non-linear evolution. The resulting angular power spectrum of temperature anisotropies is qualitatively different than the one obtained via adiabatic perturbations from an inflationary model. More precisely, one obtains a broad low peak instead of the distinct series of acoustic peaks. Moreover, string models predict generically non-Gaussianities in the CMB temperature spectrum.

The first year Planck data have been analysed [20] within the framework of $\Lambda$ CDM cosmology for a simple adiabatic model with an extra string contribution expressed in terms of the fractional contribution, $f_{10}$, of strings to the CMB temperature spectrum at multipole $\ell=10$. For an Abelian-Higgs $(\mathrm{AH})$ field theory model, the constraint turned out to be $f_{10}<0.028$, or equivalently $G \mu_{\mathrm{AH}} / c^{2}<3.2 \times 10^{-7}$ [20]. Moreover, the Planck data impose severe constraints on any primordial non-Gaussianity [21]. The evident question is whether the theoretical models can be compatible with the observational data [22].

The scalar potential in supergravity has the general form [23]

$$
V=\frac{e^{G}}{M_{\mathrm{Pl}}^{4}}\left[G_{i}\left(G^{-1}\right)_{j}^{i} G^{j}-3\right]+\frac{1}{2}\left[\operatorname{Re} f\left(\Phi_{i}\right)\right]^{-1} \sum_{a} g_{a}^{2} D_{a}^{2},
$$

where

$$
G=\frac{K}{M_{\mathrm{Pl}}^{2}}+\ln \frac{|W|^{2}}{M_{\mathrm{Pl}}^{6}}
$$

with the Kähler potential $K\left(\phi, \phi^{*}\right)$ being a real function of the scalar components of the chiral superfields $\Phi_{i}$ and their Hermitian conjugates. Upper (lower) indices $(i, j)$ denote derivatives with respect to $\phi_{i}\left(\phi^{i^{*}}\right)$, namely

$$
G^{i} \equiv \frac{\partial G}{\partial \phi_{i}}, \quad G_{j} \equiv \frac{\partial G}{\partial \phi^{j^{*}}}
$$

$f\left(\Phi_{i}\right)$ is the gauge kinetic function, and $g_{a}$ is the coupling of the $U(1)^{a}$ symmetry, which is generated by $T_{a}$ and under which the chiral superfields $S$ (with $S$ a gauge singlet playing the rôle of the inflaton), $\Phi_{+}$and $\Phi_{-}$have charges 0 , +1 and -1 respectively. Finally,

$$
D_{a}=\phi_{i}\left(T_{a}\right)^{i}{ }_{j} K^{j}+\xi_{a}
$$

where $\xi_{a}$ is the Fayet-Iliopoulos term; it can only be nonzero if $T_{a}$ generates a U(1) group.

Considering one-loop radiative corrections to the scalar potential along the inflationary valley, the effective scalar potential [24, 25] in the context of minimal supersymmetric hybrid inflation with minimal Kähler potential

$$
K=|s|^{2}+\left|\phi_{+}\right|^{2}+\left|\phi_{-}\right|^{2}
$$

lead to a large spectral index which is not preferred from the recent CMB measurements. Note that $s, \phi_{1}, \phi_{2}$ are the bosonic components of the superfields $S, \Phi_{1}, \Phi_{2}$.

However, as it has been shown in Ref. [26], supersymmetric hybrid inflation with non-minimal Kähler potential, including radiative corrections obtained through the one-loop effective potential, may lead to a red-tilted spectrum 
$\left(n_{\mathrm{s}} \approx 0.96\right)$, in agreement with the WMAP [27] and Planck [28] data. In particular, it was shown [26] that considering a non-minimal Kähler potential

$$
K=|s|^{2}+\left|\phi_{+}\right|^{2}+\left|\phi_{-}\right|^{2}+\kappa_{s} \frac{|s|^{4}}{M_{\mathrm{Pl}}^{2}}+\kappa_{s \phi_{+}} \frac{|s|^{2}\left|\phi_{+}^{2}\right|}{M_{\mathrm{Pl}}^{2}}+\kappa_{s \phi_{-}} \frac{|s|^{2}\left|\phi_{-}\right|^{2}}{M_{\mathrm{Pl}}^{2}}+\cdots,
$$

one can get a red-tilted spectrum. The consistency of this model (based on the Kähler potential Eq. (8)) with the CMB imposed constraints on the cosmic string contribution was first performed in Refs. [29, 30].

For the non-minimal Kähler potential above, Eq. (8), the effective potential reads [29]

$$
V_{\mathrm{eff}}(|s|)=\frac{g^{2} \xi^{2}}{2}\left\{1+\frac{g^{2}}{16 \pi^{2}}\left[2 \ln \left(z \frac{g^{2} \xi}{\Lambda^{2}}\right)+f_{V}(z)\right]\right\}
$$

where

$$
f_{V}(z) \equiv(z+1)^{2} \ln \left(1+\frac{1}{z}\right)+(z-1)^{2} \ln \left(1-\frac{1}{z}\right)
$$

with

$$
z \equiv \frac{\lambda^{2}|s|^{2}}{g^{2} \xi} \exp \left(\frac{|s|^{2}}{M_{\mathrm{Pl}}^{2}}+\kappa_{\mathrm{s}} \frac{|s|^{4}}{M_{\mathrm{Pl}}^{4}}\right) \frac{1}{\left(1+f_{+}\right)\left(1+f_{-}\right)}
$$

and

$$
f_{+} \equiv \kappa_{s \phi_{+}} \frac{|s|^{2}}{M_{\mathrm{Pl}}^{2}} \quad ; \quad f_{-} \equiv \kappa_{s \phi_{-}} \frac{|s|^{2}}{M_{\mathrm{Pl}}^{2}}
$$

The number of e-folds is calculated from

$$
N_{\mathrm{Q}} \equiv \ln \left(\frac{a_{\mathrm{end}}}{a_{Q}}\right)=\frac{8 \pi^{2}}{g^{2} M_{\mathrm{Pl}}^{2}} \int_{1}^{z_{Q}} \frac{\mathrm{d} z}{z^{2} f_{z}^{2}[s(z)] f_{V^{\prime}}(z)},
$$

with the index $\mathrm{Q}$ denoting the scale of the CMB quadrupole anisotropy, and the definitions

$$
\begin{aligned}
& f_{V^{\prime}}(z) \equiv(z+1) \ln \left(1+\frac{1}{z}\right)+(z-1) \ln \left(1-\frac{1}{z}\right) \\
& f_{z}(|s|) \equiv 2|S|\left[\frac{1}{M_{\mathrm{Pl}}^{2}}+\frac{2 \kappa_{\mathrm{S}}|s|^{2}}{M_{\mathrm{Pl}}^{4}}+\frac{1}{|s|^{2}}-\frac{\kappa_{s \phi_{+}}}{\left(1+f_{+}\right) M_{\mathrm{Pl}}^{2}}-\frac{\kappa_{s \phi_{-}}}{\left(1+f_{-}\right) M_{\mathrm{Pl}}^{2}}\right] .
\end{aligned}
$$

Setting the number of e-folds to about 60 , one can fix the value of the inflaton field $s_{\mathrm{Q}}$. The scalar and tensor part contributions of the inflaton field to the temperature anisotropy read [29]

$$
\begin{aligned}
\left(\frac{\delta T}{T}\right)_{\mathrm{Q}-\mathrm{scal}} & \simeq \frac{1}{4 \sqrt{45} \pi} \frac{V^{3 / 2}\left(s_{\mathrm{Q}}\right)}{M_{\mathrm{Pl}}^{3} V^{\prime}\left(s_{\mathrm{Q}}\right)} \\
& \simeq \frac{\sqrt{2} \pi}{\sqrt{45}} \frac{\xi}{g} \frac{1}{M_{\mathrm{Pl}}^{3}} z_{\mathrm{Q}}^{-1} f_{V^{\prime}}^{-1}\left(z_{\mathrm{Q}}\right) f_{z}^{-1}\left(s_{\mathrm{Q}}\right) .
\end{aligned}
$$

and

$$
\begin{aligned}
\left(\frac{\delta T}{T}\right)_{\mathrm{Q}-\text { tens }} & \simeq \frac{(0.77)}{(8 \pi)} \frac{V^{1 / 2}\left(s_{\mathrm{Q}}\right)}{M_{\mathrm{Pl}}^{2}} \\
& \simeq \frac{0.77}{8 \sqrt{2} \pi} \frac{1}{M_{\mathrm{Pl}}^{2}} g \xi
\end{aligned}
$$


respectively. The tensor over scalar ratio, $r_{\text {infl, }}$ is given by

$$
r_{\mathrm{infl}}=\frac{0.77 \sqrt{45}}{16 \pi^{2}} g^{2} z_{\mathrm{Q}} M_{\mathrm{Pl}} f_{V^{\prime}}\left(z_{\mathrm{Q}}\right) f_{z}\left(s_{\mathrm{Q}}\right)
$$

One can then compute the cosmic string contribution to the quadrupole CMB temperature anisotropy, as a function of the superpotential coupling $\lambda$, for various values of $g$ and $\kappa_{s, \phi_{ \pm}}$, which are considered as parameters. The allowed parameter space can be expressed as a constraint on the Fayet-Iliopoulos parameter $\xi$, noting that the quadrupole contribution to the CMB temperature anisotropies from the cosmic strings formed at the end of hybrid inflation is

$$
\left(\frac{\delta T}{T}\right)_{\mathrm{Q}-\mathrm{CS}} \simeq \frac{9}{4} \xi
$$

Performing a similar analysis as in Ref. [30] we obtain

$$
\sqrt{\xi} \lesssim 10^{15} \mathrm{GeV}
$$

This limit is inconsistent with the BICEP2 results [31] on the inflationary scale. Let us emphasise that computing the mass scale of symmetry breaking, given by $\sqrt{\xi}$, one finds that it increases with $\lambda$. Hence, to achieve compatibility with the inflationary scale set by BICEP2, one needs to increase the value of $\lambda$ above the upper limit (a few $\times 10^{-5}$ ) imposed from the CMB temperature anisotropies measurements.

Summarising, considering hybrid inflation with non-minimal Kähler potential one is able - through the new degrees of freedom, namely the parameters $\kappa_{\mathrm{S}}$ and $\kappa_{\phi_{ \pm}}$— to accommodate the Planck and BICEP2 data on the spectral index and the tensor-to-scalar ratio, respectively. However, the new parameters do not improve the constraint on the allowed string energy scale, which leads to a low inflationary scale. Note that the constraints will not improve by considering hybrid inflation leading to semi-local strings [32], since similar CMB constraints [33] are also imposed to such a string network. Hence, there is an inconsistency between mixed "hybrid inflation + string" models and current experimental data. To render the models compatible with the constraints one should lower the string energy scale, a trick that is no longer possible given the GUT-scale inflationary energy implied from the BICEP2 measurements, provided these measurements are indeed confirmed [34, 35]. More precisely, the BICEP2 analysis was based on dust polarization models that predicted subdominant contamination of their B-mode signal by dust polarization. Very recently, the Planck collaboration, using the Planck HFI polarization data, concluded [36] that there is a larger uncertainty in the B-modes produced by dust, implying the need for assessment of the polarized dust signal even in the cleanest windows of the sky. As a result, BICEP2 must be treated as an upper bound on primordial B-modes rather than a detection.

Turning to the brane inflationary models, one expects similar constraints on the cosmic superstring contribution to the CMB temperature anisotropies. This is due to the expected broad low peak, a generic characteristic of all models with seeds, defined as any non-uniformly distributed form of energy, which contributes only a small fraction to the total energy density of the Universe and which interacts with the cosmic fluid only gravitationally.

In short, combining the temperature anisotropies data with the results on the CMB polarisation B-modes, we unavoidably conclude that mixed "hybrid inflation + string" models face severe problems. But such models have been suggested as a realistic outcome of Grand Unified Theories applied in the context of the early Universe. Moreover, we have recently examined [37] whether supersymmetric hybrid inflation can be (naturally) embedded within the minimal SO(10) model. We have shown [37] that none of the singlets of the Standard Model symmetries in the minimal set of $\mathrm{SO}(10)$ representations, can satisfy the necessary conditions for a scalar field to play the rôle of the inflaton; a result that probably remains valid for other gauge groups beyond $\mathrm{SO}(10)$.

It is therefore clear that we are missing some important elements in order to reassemble the puzzle of the early Universe evolution. It may be worth analysing the CMB data beyond the $\Lambda$ CDM model, or further investigating inflationary models with a few e-folds after string formation [38].

[1] A. Vilenkin and P. Shellard, Cosmic strings and other topological defects (Cambridge University Press, 1994).

[2] M. Sakellariadou, Lect. Notes Phys. 718, 247 (2007) hep-th/0602276.

[3] M. Sakellariadou, Nucl. Phys. Proc. Suppl. 192-193, 68 (2009) arXiv:0902.0569 [hep-th]].

[4] A. A. Starobinsky, Phys. Lett. B 91, 99 (1980).

[5] A. H. Guth, Phys. Rev. D 23, 347 (1981). 38]

[6] A. D. Linde, Phys. Lett. B 108, 389 (1982). 
[7] A. D. Linde, Phys. Rev. D 49, 748 (1994) astro-ph/9307002].

[8] R. Jeannerot, J. Rocher and M. Sakellariadou, Phys. Rev. D 68, 103514 (2003) hep-ph/0308134.

[9] K. Dasgupta, C. Herdeiro, S. Hirano, R. Kallosh, Phys. Re[38]v. D65, 126002 (2002) hep-th/0203019].

[10] K. Dasgupta, J. P. Hsu, R. Kallosh, A. D. Linde, M. Zagermann, JHEP 0408, 030 (2004) hep-th/0405247.

[11] R. Gwyn, M. Sakellariadou and S. Sypsas, JHEP 1010, 075 (2010) arXiv:1008.0087 [hep-th]].

[12] R. Gwyn, M. Sakellariadou and S. Sypsas, JHEP 1109, 075 (2011) arXiv:1105.1784 [hep-th]].

[13] S. Kachru, R. Kallosh, A. D. Linde, J. M. Maldacena, L. P. McAllister and S. P. Trivedi, JCAP 0310, 013 (2003) arXiv:hep-th/0308055.

[14] S. Sarangi and S. H. H. Tye, Phys. Lett. B 536, 185 (2002) hep-th/0204074.

[15] G. Dvali and A. Vilenkin, JCAP 0403, 010 (2004) hep-th/0312007.

[16] J. Polchinski, Int. J. Mod. Phys. A 20, 3413 (2005) [AIP Conf. Proc. 743, 331 (2005)] hep-th/0410082.

[17] M. Sakellariadou, Phil. Trans. Roy. Soc. Lond. A 366, 2881 (2008) arXiv:0802.3379 [hep-th]].

[18] F. R. Bouchet, P. Peter, A. Riazuelo and M. Sakellariadou, Phys. Rev. D 65, 021301 (2002) astro-ph/0005022.

[19] P. A. R. Ade et al. [Planck Collaboration], arXiv:1303.5075 [astro-ph.CO].

[20] P. A. R. Ade et al. [Planck Collaboration], arXiv:1303.5085 [astro-ph.CO].

[21] P. A. R. Ade et al. [Planck Collaboration], arXiv:1303.5084 [astro-ph.CO].

[22] M. Sakellariadou, arXiv:1308.6666 [hep-th].

[23] P. Nilles, Phys. Rep. 110, 1 (1984).

[24] G. Dvali, Q. Shafi, and R. Schaefer, Phys. Rev. Lett. 73, 1886 (1994).

[25] J. Rocher and M. Sakellariadou, JCAP 0503, 004 (2005) hep-ph/0406120.

[26] M. Bastero-Gil, S. F. King and Q. Shafi, Phys. Lett. B 651 (2007) 345 hep-ph/0604198].

[27] G. Hinshaw et al. [WMAP Collaboration], Observations: Cosmological Parameter Results," Astrophys. J. Suppl. 208 (2013) 19 arXiv:1212.5226 [astro-ph.CO]].

[28] P. A. R. Ade et al. [Planck Collaboration], arXiv:1311.1657 [astro-ph.CO].

[29] J. Rocher and M. Sakellariadou, JCAP 0611, 001 (2006) hep-th/0607226].

[30] J. Rocher and M. Sakellariadou, Phys. Rev. Lett. 94, 011303 (2005) hep-ph/0412143.

[31] P. A. R. Ade et al. [BICEP2 Collaboration], arXiv:1403.3985 [astro-ph.CO].

[32] J. Urrestilla, A. Achucarro and A. C. Davis, Phys. Rev. Lett. 92, 251302 (2004) hep-th/0402032.

[33] J. Urrestilla, N. Bevis, M. Hindmarsh, M. Kunz and A. R. Liddle, JCAP 0807, 010 (2008) arXiv:0711.1842 [astro-ph]].

[34] M. J. Mortonson and U. Seljak, arXiv:1405.5857 [astro-ph.CO].

[35] R. Flauger, J. C. Hill and D. N. Spergel, JCAP 1408, 039 (2014) arXiv:1405.7351 [astro-ph.CO]].

[36] R. Adam et al. [Planck Collaboration], arXiv:1409.5738 [astro-ph.CO].

[37] G. Cacciapaglia and M. Sakellariadou, Eur. Phys. J. C 74, 2779 (2014) arXiv:1306.3242 [hep-ph]].

[38] K. Kamada, Y. Miyamoto and J. Yokoyama, JCAP 1210, 023 (2012) [arXiv:1204.3237 [astro-ph.CO]]. 